\title{
Strength of hierarchically porous ceramics: discrete simulations on X-ray nanotomography images
}

\author{
Denis Roussel ${ }^{\mathrm{a}}$, Aaron Lichtner ${ }^{\mathrm{b}}$, David Jauffrès ${ }^{\mathrm{a}}$, Julie Villanova ${ }^{\mathrm{c}}$, \\ Rajendra K. Bordia ${ }^{\mathrm{d}}$, Christophe L. Martin ${ }^{\mathrm{a}, *}$ \\ ${ }^{a}$ Univ. Grenoble Alpes, CNRS, SIMAP, F-38000 Grenoble, France \\ ${ }^{b}$ Department of Materials Science and Engineering, University of Washington, Roberts \\ Hall, Box 352120, Seattle, WA 98195, United States \\ ${ }^{c}$ ESRF The European Synchrotron, CS 40220, 38043 Grenoble Cedex 9, France \\ ${ }^{d}$ Department of Materials Science and Engineering, Clemson University, 161 Sirrine \\ Hall, Clemson, SC 29634-0971, United States
}

\begin{abstract}
Porous ceramic samples were processed either by freeze-casting or by slipcasting with pore formers. They were partially sintered to obtain a total porosity in between 44 and $69 \%$. Samples were imaged by X-ray nanotomography with $75 \mathrm{~nm}$ resolution. The images, approximately $70^{3}$ to $90^{3}$ $\mu \mathrm{m}^{3}$ in size, were merged with randomly packed particles to obtain representative numerical microstructures. The numerical samples were crushed uniaxially using discrete element simulations with appropriate microscopic fracture properties. This revealed anisotropic behavior for freeze-cast samples. Simulated strength values were compared to experimental data, with some consideration given to sample volume.

Keywords: porous material, fracture, three-dimensional tomography, discrete simulations
\end{abstract}

\footnotetext{
${ }^{*}$ Corresponding author. Email: christophe.martin@simap.grenoble-inp.fr
} 
Among the various applications requiring highly porous ceramics, filters, scaffolds for bone generation, and the electrodes in solid oxide fuel cells (SOFC) are of great engineering relevance. The functional properties of interest for these applications (permeability, bioactivity, chemical reactivity and electrical and/or thermal conductivity) are mainly dictated by the amount and morphology of their porosity. The microstructural requirements needed for optimal levels of these functional properties often contradict with the need for some required mechanical performance, especially in regards to strength $[1,2]$. Indeed, inherent brittleness and flaw sensitivity severely limit the potential use of porous ceramics. Thus, accurate methods relating strength and microstructure are needed for this important class of materials.

X-ray tomography and focused ion beam/scanning electron microscope (FIB/SEM) tomography offer the ability to reconstruct in $3 \mathrm{D}$, a representative material volume that can capture important properties. If this volume is sufficiently large, properties such as contiguity, tortuosity [3], thermal conductivity [4], elasticity [5, 6] and elastic limit [7] can be calculated. This is typically carried out by meshing the images using standard Finite Element Methods (FEM). However, starting from tomography images, fracture is a more elusive property to simulate. This is because fracture involves local topological modifications (branching, bifurcation, healing and new surface generation) that are difficult to capture even with cohesive zone models [8]. With this in mind, we show how the Discrete Element Method (DEM) can be coupled with X-ray nanotomography images of porous ceramics to compute their strength and elucidate their fracture mechanisms.

Porous ceramics for SOFC cathode application were processed using an 
aqueous composite ceramic slurry composed of a 60:40 volume ratio of yttriastabilized zirconia (YSZ, ion-conducting) and lanthanum strontium manganite (LSM, electron-conducting) powders. The slurry is obtained by mixing ceramic particles $\left(\bar{d}_{Y S Z}=300 \mathrm{~nm}\right.$ and $\left.\bar{d}_{L S M}=800 \mathrm{~nm}\right)$ into an aqueous solution containing an organic dispersant (Darvan C-N) and a binder (PEG) $[9,10]$. Two types of microstructures were processed. Directional freezecasting was used to create anisotropic samples. In this case, the slurry was poured into a cylindrical mold and cooled from one side using a temperature profile of $10^{\circ} \mathrm{C} \mathrm{min}^{-1}$ until the slurry had completely solidified (see $[9,10]$ for a detailed study of the effect of freezing rate on microstructure). Subsequent sublimation of the ice led to a porous green-body. Two values of solid loading in the initial slurry (17 and 27 vol \%) were used to produce different values of porosity after sintering. The 17 and 27 vol \% solid loadings resulted in average wall size of $15 \mu \mathrm{m}$ and $25 \mu \mathrm{m}$, respectively. For the freezing rate investigated here, the macropore size (after sintering) does not depend on solid loading and is approximately $10 \mu \mathrm{m}$.

Isotropic microstructures were produced by adding PMMA pore formers $\left(\bar{d}_{P M M A}=10 \mu \mathrm{m}\right)$ into a slurry that was then slip-cast using cylindrical molds. The size of the pore formers was selected to match the size of macropores obtained by freeze-casting (but with a narrower size dispersion). Both types of green bodies were sintered at $1200^{\circ} \mathrm{C}$ with a $2 \mathrm{~h}$ dwell time [10].

X-ray nanotomography was performed at the nano-imaging end station (ID19) of the European Synchrotron Research Facility (ESRF) to obtain 3D microstructural representations of the anisotropic and isotropic samples [11]. Four anisotropic and two isotropic samples were imaged (Fig. 1). Their 

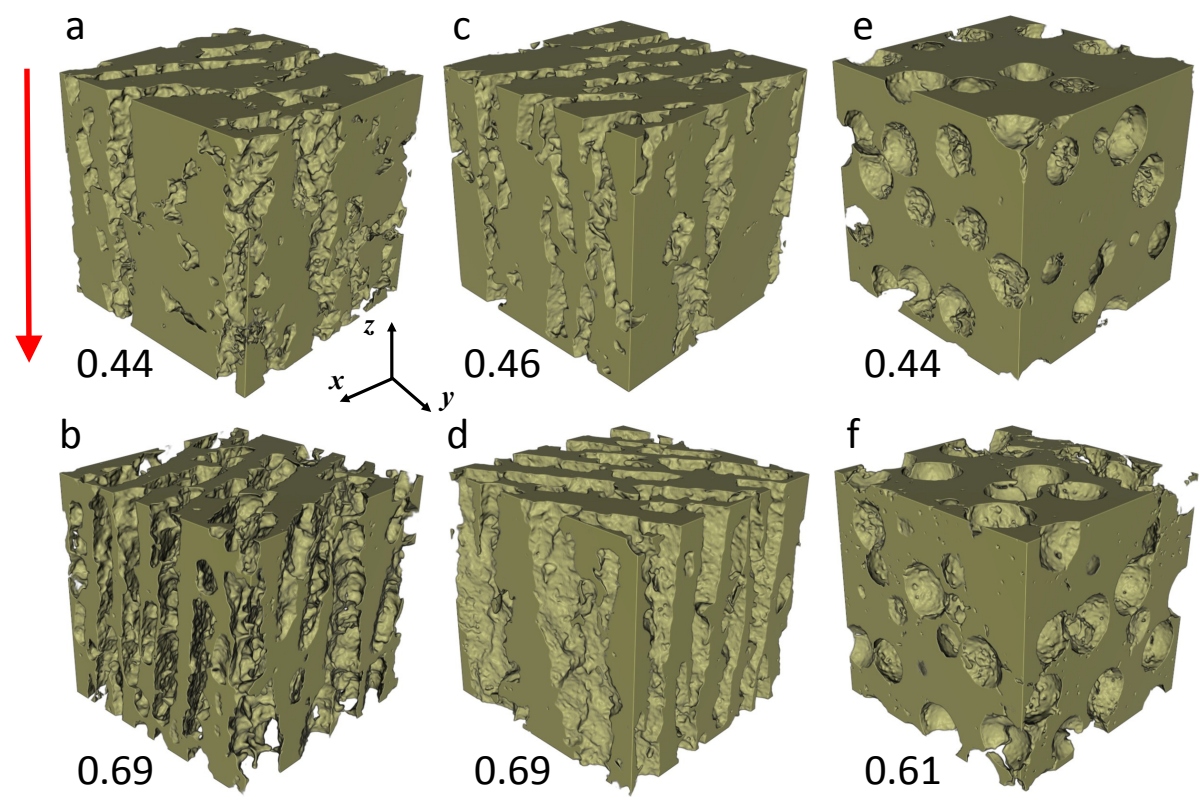

Figure 1: X-ray nanotomography images. Panels (a-d) correspond to freeze-cast samples (freezing direction indicated by the arrow); panels (e-f) to slip-cast samples. The top raw corresponds to low porosity samples $(\mathrm{a}, \mathrm{c}, \mathrm{e})$ and the bottom raw to high porosity samples $(\mathrm{b}, \mathrm{d}, \mathrm{f})$. The reconstructed volume are approximately $70^{3}$ to $90^{3} \mu \mathrm{m}^{3}$. Numbers indicate the total porosity $\epsilon$ (sum of the wall porosity and of the macroporosity in between walls for freeze-cast samples). 
total porosity levels (hereafter denoted as $\epsilon$ ), evaluated by image analysis and confirmed by Archimedes measurements, ranged between $44 \%$ and $69 \%$, depending on the solid loading in the slurry, 27 and 17\%, respectively. For freeze-cast samples, $\epsilon$ is the sum of the wall porosity and of the macroporosity in between walls (see Fig. 3 in [12]). Isotropic and anisotropic samples experienced shrinkage of the order of $20 \pm 1 \%$ and $17 \pm 2 \%$ during sintering, respectively. Anisotropic samples (Fig. 1(a-d)) exhibit large pores (macroporosity) aligned with the freezing direction $(z)$. The macroporosity in anisotropic samples is arranged in colonies at a larger scale. These colonies are typically 100 to $500 \mu \mathrm{m}$. Within these colonies, pores align with each other, leading to an additional level of architecture (Fig. 2a)). All the images of anisotropic samples were taken fully within a given colony, i.e. images do not span over several colonies.

The $75 \mathrm{~nm}$ resolution attained by X-ray nanotomography is not sufficient to discern the microstructure in the walls. However, SEM micrographs (insert in Fig. 2b) indicate that the sintering stage produced a partially sintered microstructure with porosity of the order of $25 \%$ within the walls of these ceramics [10]. Coarsening leads to YSZ and LSM particles with approximately $500 \mathrm{~nm}$ and $800 \mathrm{~nm}$ sizes, respectively.

Numerical microstructures were generated using the nanotomography images. A mixture of spherical particles of $500 \mathrm{~nm}$ and $800 \mathrm{~nm}$ diameters, representing YSZ and LSM particles, respectively, were first randomly packed into a cubical simulation box with a 60:40 volume ratio. A total of four millions of spheres were necessary to obtain a sufficiently large cube to match the scale of the nanotomography images. The numerical packing was sub- 
sequently partially sintered to attain $25 \%$ porosity and create solid bonds between particles [13]. The packed particle microstructure was then matched with the 3D nanotomography image by removing particles where the macropores were located (Fig. 2b). Note that although the average particle size and residual porosity were chosen to match the SEM observations of the solid walls, the exact location of each particle cannot be reproduced. Also, partially sintered particles are approximated as spheres indenting each other.

The solid bridges that formed during sintering are modeled as elastic bonds, whose size grows in accordance with Coble's model [14]. Bonds transmit normal and tangential forces as well as resisting moments. The elastic and fracture properties of the dense material that constitute these bonds are summarized in Table 1. In particular, it should be noted that the bond toughness is approximated as $2 \gamma_{s}$, thus representing a perfectly brittle material. Bonds may fracture in tension or shear when the stress acting on the bond exceeds the bond strength. A fractured bond does not transmit tensile forces but keeps its compressive stiffness. A Coulomb-like law (friction coefficient: $0.5)$ is utilized in shear for broken bonds. Force equilibrium is enforced to particles by repositioning each of them using a quasi-static scheme coupled with Newton's second law. A detailed description of the discrete element simulations used here may be found in [13].

The numerical microstructures were uniaxially crushed with lateral free surfaces. Freeze-cast samples exhibit anisotropic microstructures (Fig. 1) that will translate into an anisotropic response [1]. For those anisotropic samples, Fig. 2c shows that three loading directions must be tested. Two may be considered essentially equivalent from a macroscopic point of view 


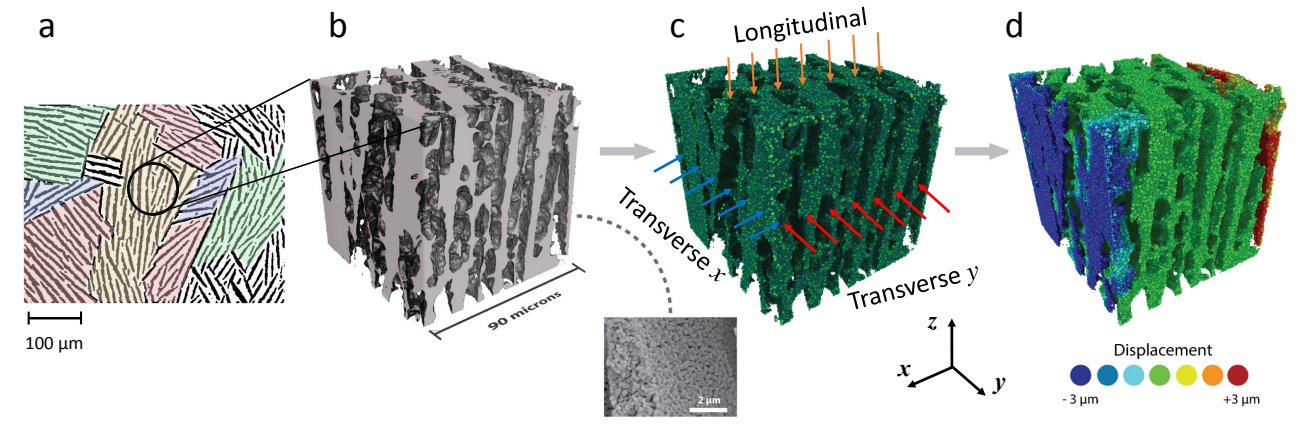

Figure 2: (a) SEM top view of a freeze-cast sample (plane perpendicular to the freezecasting direction) showing colonies of pores aligned with one another. (b-d) Generation and utilization of a numerical microstructure obtained from a nanotomagraphy reconstruction with the discrete element method. (b) X-ray nanotomography of a freeze-cast sample (sample b in Fig. 1, $\epsilon=0.69$ ). (c) Discrete microstructure matched with the 3D image. Transverse and longitudinal marks indicate the loading nomenclature. (d) Simulation of a longitudinal crushing test showing the displacement of particles in the $(x)$ direction, transverse to the loading axis $(z)$.

\begin{tabular}{cccc}
\hline & $E[15,16]$ & $\nu$ & $\gamma_{s}[17]$ \\
\hline YSZ & $220 \mathrm{MPa}$ & 0.32 & $0.5{\mathrm{~J} . \mathrm{m}^{-2}}^{-2}$ \\
\hline $\mathrm{LSM}$ & $130 \mathrm{MPa}$ & 0.32 & $0.5 \mathrm{~J} . \mathrm{m}^{-2}$ \\
\hline
\end{tabular}

Table 1: Material properties (Young's modulus, Poisson's coefficient and surface energy) of the dense particles used for simulations. 
(transverse $x$ and $y$ ), and will be denominated as transverse. However it is clear from Fig. 2c that the loading on the transverse $x$ and $y$ directions will lead to significantly different responses at the scale of the small volume tested here.

The stress-strain curves were calculated from the total reaction forces on the crushing planes and from the geometry of the crushed samples. Loadingunloading-reloading cycles were imposed to determine the material stiffness of the numerical samples during the unloading stages. On such porous microstructures, it is questionable that an elastic reversible domain exists. Thus, we prefer the term material stiffness (in units of stress) rather than elastic modulus. The macroscopic strength is also arbitrarily defined as the maximum stress attained during the crushing test (typically attained at $\approx$ $1 \%$ axial strain).

For both the anisotropic and isotropic microstructures considered here, the compressive strength and stiffness have been experimentally measured and reported in detail in [12]. Rectangular samples $(12 \mathrm{~mm} \times 12 \mathrm{~mm} \times$ $16 \mathrm{~mm}$ ) were crushed under uniaxial compression conditions [12], as in the numerical simulations. The results are reproduced in Fig. 3 for comparison. They show that isotropic samples have mechanical properties that fall in between those of anisotropic samples tested in the longitudinal and in the transverse direction. This indicates that for a given total porosity, the nature of the macro-porosity plays a significant role on mechanical properties.

Simulations on nanotomographic images lead to similar trends when compared to experimental data. For anisotropic samples loaded transverse to the freezing direction, two sets of points are shown, corresponding to the loading 


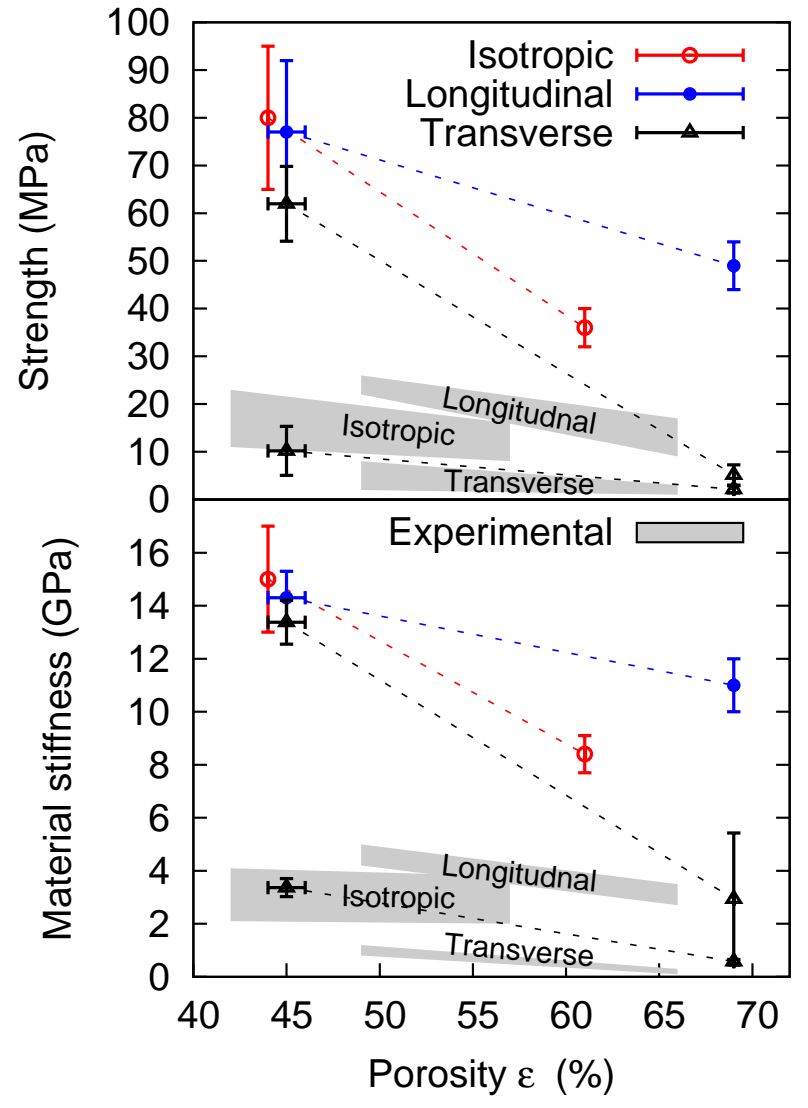

Figure 3: Strength and material stiffness from numerical tests on tomographic images compared with experimental data (shown as grey areas) [12]. Two sets of points are shown for the transversely loaded samples corresponding to the simulated loading in the $x$ (weakest response) and $y$ (strongest response) directions (Fig. 2c). 
in the $x$ and $y$ directions (Fig. 2c). Depending on the orientation of the tested colony versus the loading direction, the mechanical response is quite different. This is because in the simulations, we test a rather small volume in which a single colony is present. When considering a millimetric volume, as in the experiments, we expect the transverse mechanical response to be dictated by the colonies that are oriented normal to the loading direction. Thus, the macroscopic transverse response should be closest to the weakest response numerically tested in the transverse simulations.

Fig. 3 indicates that for samples with the largest porosity content, both strength and material stiffness are larger for anisotropic samples loaded parallel to the freezing direction (longitudinal direction) compared to transversely loaded samples. As for the experimental data, the isotropic samples exhibit a mechanical behavior approximately intermediate between the longitudinal and transversely loaded anisotropic samples. At low porosity, isotropic samples have similar strength and material stiffness values compared with anisotropic samples tested parallel to their freezing direction but fall below for large porosity content.

The simulations overestimate both strength and stiffness. The possible reasons for this are discussed below. First, we have not attempted to fit any material parameter in the model (Young's moduli and surface energies of LSM and YSZ are directly taken from literature, see table 1). Previous comparisons between numerical simulations and experimental data for partially sintered ceramics (no templated macroporosity) lead to very good agreement for elasticity [18] and reasonable agreement for strength [18, 19]. In the present case, the simulated microstructure is very close to the real one 
but the simulated volume is an issue. Classical Weibull statistics tells us that strength depends on the length of the major flaw and that the probability of failure increases with the size of the specimen. According to Weibull statistics, the probability of failure in a sample of volume $V_{1}$ is equal to that in a sample of volume $V_{2}$ if stresses $\sigma_{1}$ and $\sigma_{2}$ are related by:

$$
V_{1} \sigma_{1}^{m}=V_{2} \sigma_{2}^{m}
$$

where $m$ is the Weibull modulus that measures the scatter of strength data. Eq. (1) assumes that a single flaw type population initiates failure whatever the specimen size [20]. A full Weibull statistics analysis is out of reach with our limited data [21] and hardly applies to anisotropic samples. Also, numerous studies on porous ceramics have reported significant deviations from Weibull statistics $[22,23]$. Still, it is interesting to note that using Eq. (1) to rationalize the difference between the simulated and experimental strength leads to $m \approx 9$ for the isotropic microstructure. This value is in good agreement with the experimental data collected by Fan et al. [22] on eight different materials and more than 1560 oxide and non-oxide specimens within the porosity range studied here. Fan et al. [22] reported Weibull moduli in between 5 and 10, which are statistically independent of porosity within $10 \% \leq \epsilon \leq 55 \%$.

It is worth noting that the weakest transverse numerical strength agrees better with the macroscopic experimental data than for other conditions. This is because in that case, the simulation was actually able to approach the weakest transverse mechanical response which should dictate the macroscopic strength of the whole sample.

Eq. (1) may explain the volume dependence for the strength data. How- 

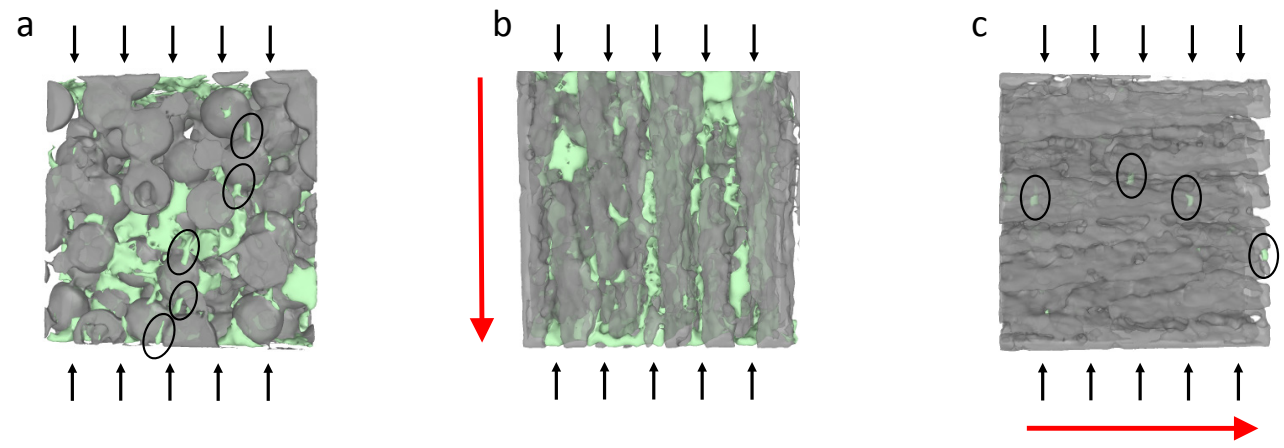

Figure 4: Fractured bonds after crushing of an (a) isotropic sample $(\epsilon=0.61)$ and of an anisotropic sample $(\epsilon=0.69)$ loaded (b) parallel and (c) transverse to the freezing direction (red arrow). Macropores are light gray and clusters of fractured bonds are green.

ever, in order to explain the volume dependence of material stiffness it is required to assume that some fracture events also occur very early on during the deformation of samples. Those early damage events affect the material stiffness and would help to explain its dependence on volume. This idea is well corroborated by our observation that in the simulations, although bonds break predominantly just before the stress maximum is attained, some bonds do break quite early. This indicates that for such high porosity materials, it is not possible to define a purely elastic early stage regime.

Fracture mechanisms can be investigated at the particle length scale using discrete simulations. Fig. 4 shows the location of fractured bonds together with large pores right after the stress maximum. The isotropic sample (Fig. 4a) exhibits a rather heterogeneous distribution of broken bonds, with heavily damaged zones, surrounded by nearly intact ones. We observed that the initial damage spreads as cracks. The crack segments depicted by ellipses in Fig. 4a pertain to the same crack that has grown early in the fracture pro- 
cess. Anisotropic samples tested parallel to the freezing direction (Fig. 4b) show damage homogeneously located in the walls. In these samples, damage has expanded across the whole width of the ceramic wall. Conversely, Fig. 4c indicates that a very small amount of damage is sufficient to crush the sample when the ceramic walls are oriented transverse to the loading direction. Note that this sample $(\epsilon \approx 0.69$, Fig. $1 \mathrm{~b})$ had an extremely low strength value (1.4 MPa), as expected. The macroscopic strength for transversely loaded samples should be dictated by colonies that are oriented perpendicular to the loading direction and which exhibit the lowest strength (Fig. 3). Further gains in mechanical performance of anisotropic freeze-casts should be realized by optimization of strength of those colonies. In any case, we have shown here that for freeze-cast materials, one should not focus solely on properties in the direction of freezing.

The possibilities offered by coupling X-ray (nano)-tomographic images and the Discrete Element Method (DEM) have been demonstrated here. It should be clear that DEM is not the only appropriate method for simulating elasticity from 3D images. The finite element method, supported by decades of research on the subject, is also a very effective method. However, when fracture is considered, DEM may be an interesting option. The topological modifications that come with fracture are easier to capture with DEM than with the finite element method. Although, as exemplified here, the imaged volume may be insufficiently large to account for the macroscopic fracture properties, this type of simulation should give valuable insights on the fracture mechanisms of porous materials at the length scale of particles. 
The authors thank the National Science Foundation (NSF, grant no. 1008600) and the French Agence Nationale de la Recherche (ANR, grant no. 2010 BLAM 0931 01).

[1] T. Ohji, M. Fukushima, International Materials Reviews 57 (2) (2012) $115-131$.

[2] S. Meille, M. Lombardi, J. Chevalier, L. Montanaro, Journal of the European Ceramic Society 32 (15) (2012) 3959-3967.

[3] J. Laurencin, R. Quey, G. Delette, H. Suhonen, P. Cloetens, P. Bleuet, J. Power Sources 198 (2012) 182-189.

[4] S. Grandjean, J. Absi, D. S. Smith, J. Eur. Ceram. Soc. 26 (2006) 26692676.

[5] A. P. Roberts, E. J. Garboczi, J. Am. Ceram. Soc. 83 (2000) 3041-3048.

[6] Z. Chen, X. Wang, F. Giuliani, A. Atkinson, Acta Materialia 89 (2015) 268-277.

[7] L. Zhang, J. M. F. Ferreira, S. Olhero, L. Courtois, T. Zhang, E. Maire, J. C. Rauhe, Acta Mater. 60 (2012) 4235-4246.

[8] J. Planas, M. Elices, G. V. Guinea, F. J. Gómez, D. a. Cendón, I. Arbilla, Engineering Fracture Mechanics 70 (14) (2003) 1759-1776.

[9] A. Z. Lichtner, D. Jauffrès, C. L. Martin, R. K. Bordia, J. Am. Ceram. Soc. 96 (9) (2013) 2745-2753. 
[10] A. Z. Lichtner, D. Jauffrès, D. Roussel, F. Charlot, C. L. Martin, R. K. Bordia, Journal of the European Ceramic Society 35 (2) (2015) 585-595.

[11] J. Villanova, P. Cloetens, H. Suhonen, J. Laurencin, F. Usseglio-Viretta, E. Lay, G. Delette, P. Bleuet, D. Jauffrès, D. Roussel, A. Z. Lichtner, C. L. Martin, Journal of Materials Science 49 (16) (2014) 5626-5634.

[12] A. Lichtner, D. Roussel, C. L. Martin, R. K. Bordia, Journal of the American Ceramic Society (2015) in press.

[13] D. Jauffrès, C. L. Martin, A. Lichtner, R. K. Bordia, Acta Mater. 60 (2012) 4685-4694.

[14] R. L. Coble, J. Am. Ceram. Soc. 41 (1958) 55-62.

[15] S. Giraud, J. Canel, J. Eur. Ceram. Soc. 28 (2008) 77-83.

[16] A. Selçuk, A. Atkinson, J. Eur. Ceram. Soc. 17 (1997) 1523-1532.

[17] C.-W. Kuo, Y.-H. Lee, K.-Z. Fung, M.-C. Wang, J. Non-Cryst. Solids 351 (2005) 304-311.

[18] X. Liu, C. L. Martin, D. Bouvard, S. D. Iorio, J. Laurencin, G. Delette, J. Am. Ceram. Soc. 94 (2011) 3500-3508.

[19] D. Jauffrès, X. Liu, C. L. Martin, Engineering Fracture Mechanics 103 (2013) 132-140.

[20] R. Danzer, T. Lube, P. Supancic, R. Damani, Adv. Eng. Mater. 10 (2008) 275-298. 
[21] S. Matsuda, M. Takahashi, Engineering Fracture Mechanics 77 (2010) 2601-2609.

[22] X. Fan, E. D. Case, F. Ren, Y. Shu, M. J. Baumann, Journal of the mechanical behavior of biomedical materials 8 (2012) 21-36.

[23] O. Keleş, R. E. García, K. J. Bowman, Acta Materialia 61 (19) (2013) $7207-7215$. 
